\title{
化学之旅一一博物馆奇游记
}

杨火青, 龚钰扉, 吕七, 张立春 ${ }^{*}$

四川大学化学学院, 成都 610064

摘要: “经黑浊、黄白、青白, 待炉火纯青之时, 方可铸器”, 从器物的诞生, 到材质的演变, 无一不是化学魅力 的彰显。本文以日记的形式, 记述了一段我在梦中与陶瓷、青铜器、铁器相遇、相知、相熟的奇妙旅程, 旨在让读 者在轻松活泼的故事中，去感悟那些逐渐被人们所淡忘的国宝背后所蕴藏的化学知识。

关键词: 博物馆; 国宝; 陶瓷; 青铜器; 铁器; 科普

中图分类号: G64; O6

\section{Chemical Tours: A Curious Journey to the Museum}

\section{Huoqing Yang, Yufei Gong, Yi Lü, Lichun Zhang *}

College of Chemistry, Sichuan University, Chengdu 610064, P. R. China.

Abstract: "When the fire is pure blue, cast can begin", from the birth of the ware to the evolution of the material, it is all about the fascination of chemistry. In the form of a diary, this article recounts a fascinating journey in which I met, got to know and became acquainted with ceramics, bronze and iron in my dreams, aiming at making the reader realize the chemical knowledge behind those gradually forgotten national treasures in a relaxing and lively story.

Key Words: Museum; National treasure; Ceramic; Bronze; Ironware; Popularization of science

我是一名普普通通的大学生, 专业为化学, 成绩很一般。每当遭受挫折时, 我总是归处于自己 不够聪明, 注定一生碌碌无为, 于是就放弃了努力, 直到今天一切都悄然改变.......

今天是周末, 父母硬要带我来参观博物馆, 我坚决反对, 却还是被拖了过来。没逛多久, 我便 觉得无聊, 于是假装上则所, 偷偷跑到了一个小角落, 掏出手机开始玩了起来。可是不一会儿, 一 阵困意袭来，我便倚靠在墙边睡着了。

\section{1 陶和瓷的重逢}

我迷迷糊糊地从梦中醒来, 发现依旧身处博物馆, 只是周围没有一点人的痕迹。这时, 前面的 展览室突然传来 “咚咚咚” 的声音。虽然有些害怕, 但我还是走了过去, 发现一个像人头的罐子从 展览柜中蹦了出来!

我吃惊地大叫道: “什么东西? 还能自己动?”

收稿: 2020-08-12; 录用: 2020-09-07; 网络发表: 2020-09-18

“通讯作者, Email: zhanglichun@scu.edu.cn

基金资助：四川大学创新火花项目(2018SCUH0078); 四川大学新世纪高等教育教学改革工程第八期(SCU8157) 
他将脸转了过来，回复道: “我是仰韶文化时期的文物一一头壸(图 1)。”

我发觉他居然会说话, 更为吃惊了, 转身就准备跑, 却听到身后再次响起人头䡬的声音: “我不 过就是一个会说话的陶器而已, 你不用太害怕我, 我并无恶意。”

我转过头, 再次打量了他一圈, 他就像用泥塑成的一般, 周身都是粗粘的, 似乎真的没有可威 胁到我的地方。我稍微放松了警惕, 疑惑地问道: “倘若我没记错, 陶器应是以黏土或者陶土为原材 料, 通过纯手工塑形，再经烧制而成？但是黏土和陶土具体是什么，我却记不太清楚了。”

人头壶回答道: “你说得没错, 黏土就是硅酸盐矿物历经无数个日夜而得以形成的, 至于陶土则 是一个庞大的家族, $\mathrm{Al}_{2} \mathrm{O}_{3} 、 \mathrm{SiO}_{2} 、 \mathrm{Fe}_{2} \mathrm{O}_{3}$ 便是其中最主要的三支。回溯千年之前的仰韶文化时期, 人类便足以凭借双手创造各种东西, 陶碗、陶罐、陶锅……我也可算得上其中的杰出代表了!”

我有些迷惑地问道: “那你是怎么制作而成的？为何周身是通红的呢？”

这话仿佛勾起了他的回忆, 人头壶沉思了一会儿, 开始解释道: “我属于陶器中的红陶, 我还有 好几个兄弟姐妹一一灰陶、黑陶和白陶(图 2)。我是他们中最年长的, 当我出生时, 人们对于窝室气 密性的控制还不够成熟, 因此有大量的空气进入, 而空气中含有 $21 \%$ 的氧气[1], 过量的氧气将陶胎 中的 $\mathrm{FeO}$ (黑色)和 $\mathrm{Fe}_{3} \mathrm{O}_{4}$ (黑色)全部氧化成 $\mathrm{Fe}_{2} \mathrm{O}_{3}$ (红色), 于是呈现红色; 而到二弟灰陶降生之时, 人们已经发明了封闭㝨顶, 在制作时堵死出气孔后向㝨室内喷水, 以减少氧气量并带走部分热量, 此时燃料的不充分燃烧所产生的 $\mathrm{CO}$, 将部分 $\mathrm{Fe}_{2} \mathrm{O}_{3}$ 还原为 $\mathrm{Fe}_{3} \mathrm{O}_{4} 、 \mathrm{FeO}$, 从而使陶器呈现灰色; 三 弟黑陶似乎就不太幸运了, 人们大量燃烧柴草, 所产生的黑烟(炭黑)升起后, 均匀地渗入陶胎, 故而 呈现黑色 ${ }^{[2]}$; 而四妹白陶是我们当中最为纯洁的, 这源于她制作时所用原料一般为高岭土和高铝粘 土, 其中 $\mathrm{Al}_{2} \mathrm{O}_{3}$ (白色)的量远大于 $\mathrm{Fe}_{2} \mathrm{O}_{3}$ (红色) ${ }^{[3] \ldots . . . ”}$

人头壶的话还没有说完, 一个青色的影子(图 3)闪现而出, 甜美的声音也随之传来: “人头壶大 哥, 好久不见! 现在被人类称为 ‘陶瓷” 的我们, 时别几百年, 再次重逢了呢!”



图 1 人头壶 ${ }^{[4]}$

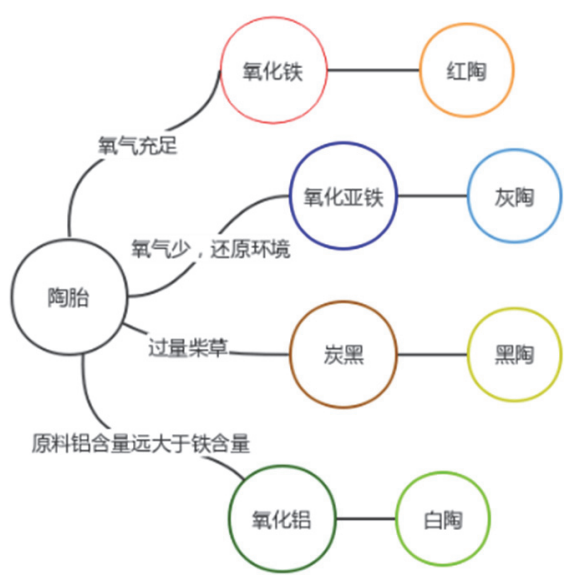

图 2 陶器制作过程

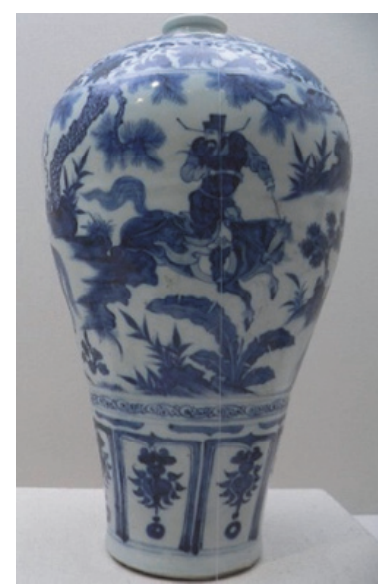

图 3 青花瓷 ${ }^{[5]}$

我聚焦于那全身覆有精美花纹的青色身影, 吃惊地问道: “莫非你是青花瓷? 你们居然相互认 识, 那你们 “陶” 和 “瓷” 具体有什么区别呢? ”

青花瓷回答道: “没错, 我就是出于景德镇窑的青花梅瓶, 和人头壶哥哥是老朋友了, 他早于我 几千年诞生, 可以称得上是我的老师了。瓷和陶的不同之处在于: 瓷器的原料中掺入了高岭土 $\left(\mathrm{Al}_{2}\left(\mathrm{Si}_{2} \mathrm{O}_{5}\right)(\mathrm{OH})_{4}\right)$, 它不仅造就了瓷器洁白无瑕, 更使得瓷器的可塑性和耐热性得到了极大的提高。 景德镇也因其周围富含大量的高岭土, 使得瓷器产业得以兴旺发展。值得一提的是, 瓷器的表面还 覆有华丽的青色图案以及一件透明的衣服——釉。”

听她那么一说, 我很是感兴趣, 急切地问道: “这青色的图案如此的光彩夺目, 它是怎么形成的 
呢?”

青花瓷继续回答道: “色彩源于氧化钴(黑色), 你们人类现在也在使用 $\mathrm{CoO}$ 与 $\mathrm{SiO}_{2} 、 \mathrm{Al}_{2} \mathrm{O}_{3}$ 或者 $\mathrm{ZnO}$ 反应制得的颜料吧 ${ }^{[6]}$ 。景德镇不仅开采高岭土, 同时还发掘钴矿料。由于天然钴矿的钴含量最 多达到 5\%, 故需要通过加工方能制成青花料。专业的匠师在干燥后的瓷胚上用青花料绘就美丽的图 画, 再在表面施以一层透明釉, 于窝中烘制即可(图 4)。烘烤过程中温度达到 $1200{ }^{\circ} \mathrm{C}$, 青花料中的 氧化钴与釉中的二氧化硅等物质发生了复杂的化学反应, 生成了蓝色的含钴物质。因此如果不施釉、 温度不够或者青花料的制备出现了问题，都将得不到如此惊艳的青花瓷了!”



图 4 瓷器制作过程

我试着用手触摸了一下青花瓷的表面, 发觉其竟然无比的光滑, 不禁感叹道: “这难道就是釉? 太神奇了吧, 就像玻璃一样。”

青花瓷回答道: “你说得没错, 那其实是我的防护服。其实釉也没有什么特别的, 其原料和瓷胚 大体是差不多的, 只是加入了一些特殊的矿物和助熔剂, 以使其更容易熔融。在烘烤时, 已完全化 为液体的釉料在表面张力的作用下均匀地覆盖在瓷体表面, 待冷却后, 便凝固成像玻璃的物质 ${ }^{[7]}$ 。 釉不仅让我们拥有了绚丽的衣服，同时还让我们拥有了更加强壮的身体以及不惧衰老的容颜。”

……后人头壶和青花瓷开始谈论起他们从前的往事, 此时我才发觉我和父母走失了, 在依依 不舍地向他们告别后, 我朝着出口走去, 没想到却来到了另外一个展览室。

\section{2 青铜器结义}

这个展览室比上一个更大, 中心的一个庞然大物(图 5)吸引了我 的目光, 就在这时它威严的声音骤然响起: “人类你好, 我是殷墟出 土的司母戊鼎, 目前已知最大、最重的青铜器王者。”

我被司母戊鼎的声音所震慑, 大脑一片空白: “青铜? 何以不是 纯铜器呢？难道古代人没法提纯？”

司母戊鼎浑厚的声音再次传来: “非也, 我们青铜作为一种合金, 是在纯铜之中掺入了锡、铅等金属元素 ${ }^{[8]}$ 。虽然我们经常被纯铜嘲 笑为 “不够纯正”, 但是我们却比其更加强壮、长寿, 并且在更低的 温度下便可锻造。殷墟时期的青铜器不仅种类和样式繁多, 而且其 上的花纹更是华丽。”

我凝视着司母戊鼎身上的花纹, 突然心生疑惑, 问道: “你历经 时间千年的洗礼, 身上为何没有明显的锈迹? ”

司母戊鼎显得有些激动, 回答道: “其实我们青铜一族在保存的 时候都会或多或少生锈, 这是由于铜、锡的氧化物或者氯化物比单

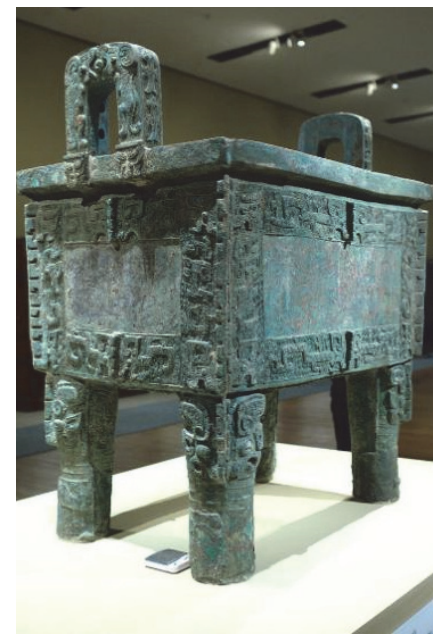

图 5 司母戊鼎 ${ }^{[9]}$ 质更加稳定。但在出土之后, 仰仗于你们人类的悉心照顾, 我们才得以如此风光。我身上原本的锈 可简单地分为两种一一无害锈与有害锈(图 6)。无害锈主要成分是: $\mathrm{CuO}$ (黑色)、 $\mathrm{Cu}_{2} \mathrm{O}$ (红色)、 $\mathrm{Cu}_{2}(\mathrm{OH})_{2} \mathrm{CO}_{3}$ (绿色)、 $\mathrm{CuS}$ (黑色)等, 这些物质在青铜表面形成保护层, 不仅在一定程度上保护了我 们, 而且使我们的色彩更加丰富; 而有害锈的主要成分为: $\mathrm{CuCl}$ (白色)和 $\mathrm{Cu}_{2}(\mathrm{OH})_{3} \mathrm{Cl}$ (墨绿色), 其 中后者就是 “青铜病” 的罪魁祸首, 它们不仅会使青铜自身整体粉化, 以至于完全毁坏, 还能 “传 染, 给其他的青铜 $[10]$, 可以说是罪蒘深重呀!” 


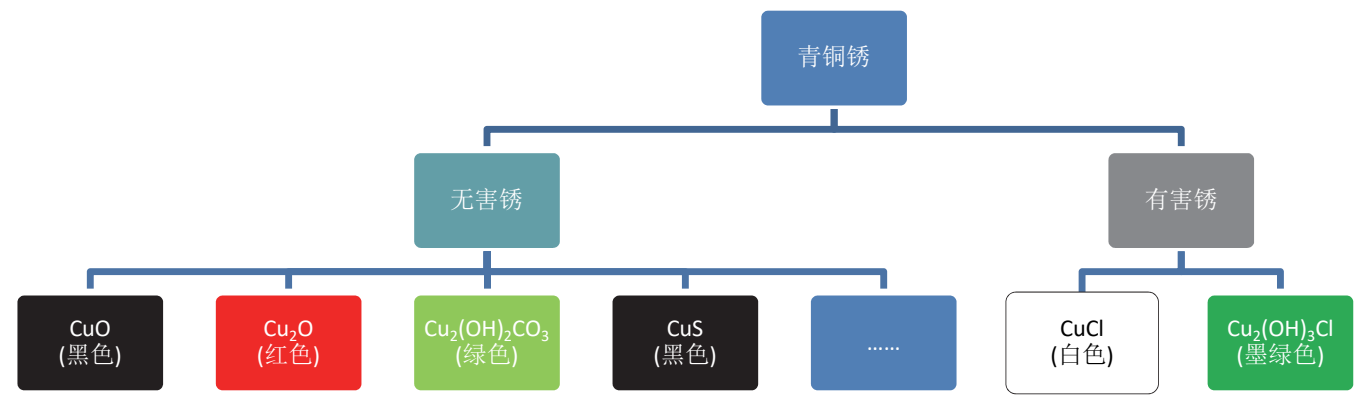

图 6 青铜锈的主要成分

我急忙问道: “那如何才能根除 “青铜病” 呢?”

司母戊鼎回答道: “除锈的方式很多, 有物理除锈法、化学除锈法, 其中我记得比较清楚的一种 是倍半碳酸钠法: 倍半碳酸钠溶液就是 $\mathrm{Na}_{2} \mathrm{CO}_{3}$ 和 $\mathrm{NaHCO}_{3}$ 的混合水溶液 ${ }^{[11]}$, 用一定浓度的倍半碳 酸钠溶液浸泡青铜器, 由于在溶液中碳酸铜的溶解度小于氯化铜, 所以优先生成, 因此锈中的氯离 子就被置换出来了, 通过不停地更换新的倍半碳酸钠溶液, 直到溶液中氯离子浓度小于一定值时, 除锈就算完成了。”

我又将目光转移到司母戊鼎的介绍牌上: “高 133 厘米、口长 112 厘米、口宽 79.2 厘米, 重 832.84 公斤! 你如此庞大沉重, 是怎么制成的呀? ”

司母戊鼎自豪地回答道: “先不说方法, 我的诞生, 可是在两三百名工匠的共同努力下, 耗费了 至少 1000 公斤的原料才得以完成的。至于方法, 我记得好像被现代人称为 “范铸法”, 即先建模、 再制范、最后铸器!”

我突然回想起青铜器的相关知识, 但又有点模糊: “我记得 “模” 和 “范” 都是为了给器具塑形, 那他们有什么区别呢? ”

司母戊鼎回答道: “你说的没错, 区别在于, 建 '模, 是为了制 ‘范', 而 “范” 才是直接铸器 的工具。“模” 只是泥经前处理、造型、刻图、晾干、烧制后而形成的中间材料。而 “范” 是在 “模” 的轮廓上, 用一定比例的土、砂、植物纤维、草木灰、熟料等混合物进行覆盖, 再经由焙烧得以成 型。在 '范' 中加入砂是为了能够承受更高的温度, 而加入植物纤维和草木灰则是为了防止气泡残 存于器内 ${ }^{[12]}$, 总而言之, “范” 比 “模” 更为强壮, 并且是青铜器的直接导师。我当时便是在 28 位 老师的共同指导下，应运而生的。”

一个成语突然闪入我的脑海, 我脱口而出: “我记得 “炉火纯青” 就是源于青铜器的炼制？”

司母戊鼎肯定地回答道: “确实如此, “凡铸金之状, 金与锡。黑浊之气竭, 黄白次之; 黄白之 气竭, 青白次之; 青白之气竭, 青气次之; 然后可铸也。” 金即为铜, 在铸造青铜时, 会依次出现黑 色、黄白色、青白色、青色的火焰, 而只有当青色的火焰出现时, 才可铸器(图 7)。”



图 7 青铜炼制过程 
我好奇地问道: “火焰为什么会有这么多种颜色呀?”

司母戊鼎笑了笑回答道: “那是因为, 原料并非纯铜, 随着温度的升高, 其中所含有的各种杂质, 通过挥发或者升华, 进入火焰。最开始的黑色火焰是源于铜中有机物的燃烧, 当有机物含碳量较高 且氧气不充分时, 燃烧就会产生极细的碳粉, 即炭黑或称之为碳烟; 而黄白色火焰是因为熔点低的 锡熔化后与铜中的氧化物、硫化物等杂质一同混入火焰而产生的; 至于青白色火焰则是由于部分铜 熔化造成的。”

我急忙追问道: “为什么铜熔化时，火焰颜色会变成青色？我记得原来做铜的焰色反应的时候, 看到的火焰是绿色的呀。”

司母戊鼎睿智地点了点头, 说道: “这个问题很好, 简而言之, 你实验所看到的绿色火焰是由 $\mathrm{Cu}(\mathrm{II})$ 产生的, 而青色的火焰是由 $\mathrm{Cu}(\mathrm{I})$ 产生的, 至于为何如此, 便要从在不同状态下铜以何种价态 存在更稳定说起了。铜原子的价电子排布为 $3 d^{10} 4 s^{1}$, 按理说失去一个电子的 $\mathrm{Cu}(\mathrm{I})$ 更加稳定, 但为何 在我们生活中铜离子大多以 $\mathrm{Cu}(\mathrm{II})$ 的形式存在呢, 这是因为 $\mathrm{Cu}(\mathrm{II})$ 电荷数更高, 水合热更大, 在溶液 中 $\mathrm{Cu}(\mathrm{II})$ 更稳定, 但是在干态或者高温时, 相较于 $\mathrm{Cu}(\mathrm{II}), \mathrm{Cu}(\mathrm{I})$ 明显更为稳定, 其焰色反应则为青色 (浅蓝色)。”

他的话令我醍醐灌顶, 我不禁赞叹道: “青铜器王者果然博文广识呀!”

司母戊鼎自豪地笑道: “哈哈！过奖了，其实我……”

就在这时，一个更为高大的青色影子(图 8)闪现而出，空灵的声 音幽幽地传来: “久闻司母戊鼎的大名，今日一见，果然名不虚传呀！ 我是青铜神树, 高 3.95 米, 有三簇树权, 每簇有三根树枝, 每枝上 有二十七颗果实和九只小鸟, 意为 “九日居下枝”, 被人们昵称为 “摇 钱树”, 更有“登天之梯”之美誉。”

我凝视着她那精致的外形, 不禁发问: “如此复杂的结构, 是如 何铸造而成的呀?”

青铜神树解释道: “司母戊鼎和我在铸造上有所不同, 由于他没 有复杂的结构, 所以只需一次铸造即可成型, 而我需要分段铸造才可 制成, 并且还运用了套铸、铆铸等技术。”

……他们也开始叙旧了, 我突然想起还得赶快与父母会合, 急忙 寻找着新的出口。这时一阵凌厉的剑气袭来, 令我不寒而栗, 随之而 来的是一句充满锐气的话: “人类! 先别走, 我还有话要说!”

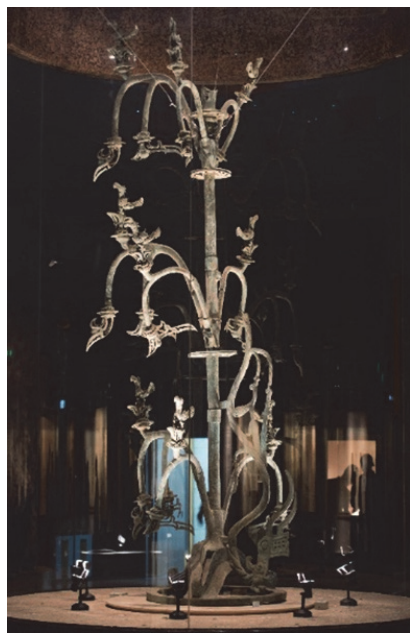

图 8 青铜神树 [13]

定睛一看, 说话的是一把小巧的利剑(图 9), 此时司母戊鼎和青 铜神树的叙旧也戛然而止, 于是他接着说道: “司母戊鼎、青铜神树, 二位前辈, 久仰大名! 我是青 铜器 ‘越王勾践剑”, 被誉为 “天下第一剑”。刚出土的我, 光洁如新、锋利无比, 剑身由铜、锡、 铅、铁等元素构筑而成, 护手处有蓝色琉璃和绿松石点缀, 非常高兴能在这里与你们相遇! ”说完便 朝司母戊鼎和青铜神树走去，他们三个互相致意问好。

我察觉到越王勾践剑光洁如新、锋利无比背后的不同寻常, 不禁问道: “青铜器“千年不锈”者, 可谓风毛麟角, 你是如何做到的? ”

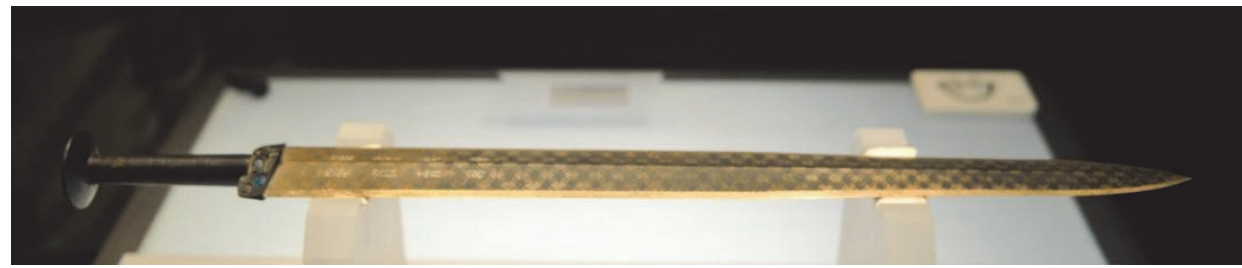

图 9 越王勾践剑[14] 
越王勾践剑甚是自豪: “首先你需要知道青铜为什么会生锈, 由方程式(1)-(4)可知生锈的主要原 因是空气和水的侵入 ${ }^{[15]}$ 。我由 $80 \%$ 的铜、 $16 \%-17 \%$ 的锡, 以及少量的铝、铁、镍组成, 而作为主要

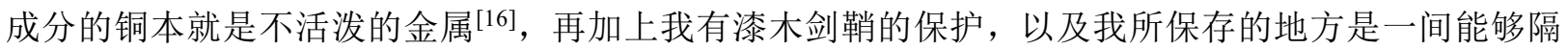
绝水和空气的密室 ${ }^{[17]}$, 我便得以保存得如此完美, 以至于 ‘千年不锈” ......”

$$
\begin{aligned}
& \mathrm{Cu}+\mathrm{Cl}^{-} \rightarrow \mathrm{CuCl}+\mathrm{e}^{-} \\
& 2 \mathrm{CuCl}+\mathrm{H}_{2} \mathrm{O} \leftrightarrow \mathrm{Cu}_{2} \mathrm{O}+2 \mathrm{HCl} \\
& \mathrm{Cu}_{2} \mathrm{O}+\mathrm{H}_{2} \mathrm{O}+\mathrm{CO}_{2} \rightarrow \mathrm{CuCO}_{3} \cdot \mathrm{Cu}(\mathrm{OH})_{2} \\
& 4 \mathrm{CuCl}+4 \mathrm{H}_{2} \mathrm{O}+\mathrm{O}_{2} \rightarrow \mathrm{CuCl}_{2} \cdot 3 \mathrm{Cu}(\mathrm{OH})_{2}+2 \mathrm{HCl}
\end{aligned}
$$

\section{3 铁器的思考}

““天下第一剑”, 让我们来切磋一下吧!”话音未落, 一道更为凌厉的剑气从我的脸庞快速划过, “铭!” 那不速之客便与越王勾践剑撞在一起, 发出了巨大的声音。我随声望去, 发现是一把与越王 勾践剑长度差不多的剑(图 10), 虽然没有华丽的装束, 但却散发着炫目的寒光。在两把剑的较量中, 后者似略胜一筹, 相比于越王勾践剑, 它更灵活, 也更有劲。几十个回合之后, 越王勾践剑有些招 架不住了, “哐当!”随着一声巨响, 两把宝剑分开了。



图 10 龙泉剑[18]

稍微站定之后, 后者率先开口: “不愧是 ‘天下第一剑”, 由青铜材料制成, 居然也可以如此厉 害!”

越王勾践剑边喘边说道: “过奖了，你是何方神圣？竟更胜我一筹。”

后者回答道: “不同于你们, 我是一把铁剑, 而且是第一把铁剑, 名为 “龙泉”, 代表着诚信与 高洁。”

龙泉剑剑身迸发而出的炫目寒光让我尤为惊讶，我惊奇地问道：“你为什么会寒光四射？”

龙泉剑用低沉的声音回答道: “这便是 ‘金属光泽”，简而言之，就是当光照在某些金属上时， 其中的可见光被全部反射, 因此金属呈现白色。具体来说就是, 金属的价层电子极易失去, 游离于 金属晶体内部各个原子之间, 因此整个晶体就相当于一个等离子体, 而每种等离子体都有一个固有 的等离子频率, 它只与等离子体中的自由电子密度有关, 只有当光的频率大于或者等于这个固有频 率时才能被吸收, 否则就会被反射, 多数金属的等离子体频率相当高, 基本达到紫外线波段, 故频 率较低的可见光就会被金属全部反射。”

我又追问道: “那为什么切碎的金属，会呈现黑色呢？”

龙泉剑笑答道: “也会有金属光泽的, 只是需要更加微观的视角而已, 比如说用你们人类发明的 显微镜观察。”

我突然又有了一个疑问: “青铜器的出现远早于铁器, 但其需求量却远不如铁, 这是为什么呢? ” 
越王勾践剑有些不开心地说道: “他们的身体比我们更为硬朗, 并且来源也更为丰富。我们唯一 的优点可能就是没有他们容易生锈。唉, 这样一对比, 突然觉得我们青铜一族似乎没有什么存在的 意义了, 怪不得铁器出现后我们就被人类遗弃了……”

龙泉剑看出了越王勾践剑的消沉之意，悄悄来到他的身边，拍了拍他的肩膀说道: “青铜剑兄， 不要这么想。有比你强的剑存在, 你就没有意义了吗? 那可未必。你被人们提起时, 他们关注的并 不是你有多锋利之类的, 而是你代表着 “吃得苦中苦, 方为人上人' 的人生哲理, 你的价值远超你 所见的, 因为你的每一分努力都会使你的价值得以增长; 而我的价值便是 '开拓创新, 不惧质疑', 无人用铁铸剑, 由我来开这个先河, 虽然有很多质疑, 但是我深知 “唯有创新, 才会进步, 所以我 坚持了下来…...”

\section{4 梦醒时分}

父母的呼喊将我惊醒，我似乎做了一场梦，又似乎没有，但有一点是确定的，我的脑海里多了 一个挥之不去的声音: “也许我永不会是最优秀的, 但是我也不能放弃努力, 即使无法成为最强者, 我也不能松解或者气馁, 因为努力是会有收获的, 哪怕失败也要比不去尝试更好, 有位伟人曾说过 “向前进总要比站在原地更加幸福’, 便是如此!”

跟着父母继续游览博物馆, 馆中的人头壶、青花瓷、司母戊鼎、青铜神树、越王勾践剑、龙泉 剑突然显得十分亲切……

\section{参 考 文 献}

[1] 空气. [2020-08-21]. https://zh.wikipedia.org/wiki/\%E7\%A9\%BA\%E6\%B0\%94.

[2] 周嘉华, 赵匡华. 中国化学史(古代卷). 南宁: 广西教育出版社, 2003: 71.

[3] 周嘉华, 赵匡华. 中国化学史(古代卷). 南宁: 广西教育出版社, 2003: 75 .

[4] 新石器时代红陶人头壸. [2020-08-07].

https://bkso.baidu.com/item/\%E6\%96\%B0\%E7\%9F\%B3\%E5\%99\%A8\%E6\%97\%B6\%E4\%BB\%A3\%E7\%BA\%A2\%E9\%99\%B6\%E4\%BA\%B $\mathrm{A} \% \mathrm{E} 5 \% \mathrm{~A} 4 \% \mathrm{~B} 4 \% \mathrm{E} 5 \% \mathrm{~A} 3 \% \mathrm{~B} 6 / 23649044$ fromtitle=\%E4\%BA\%BA\%E5\%A4\%B4\%E5\%A3\%B6\&fromid=22311713.

[5] 青花瓷. [2020-08-08]. https://zh.wikipedia.org/wiki/\%E9\%9D\%92\%E8\%8A\%B1\%E7\%93\%B7.

[6] 氧化钴. [2020-08-19]. https://baike.baidu.com/item/\%E6\%B0\%A7\%E5\%8C\%96\%E9\%92\%B4.

[7] 釉. [2020-08-19]. https://zh.wikipedia.org/wiki/\%E9\%87\%89.

[8] 魏珍. 不同地区埋藏环境土壤特征与出土青铜器锈蚀之间的关系[D]. 西安: 西北大学, 2008 .

[9] 司母戊鼎. [2020-08-09]. https://zh.wikipedia.org/wiki/\%E5\%8F\%B8\%E6\%AF\%8D\%E6\%88\%8A\%E9\%BC\%8E.

[10] 徐群杰, 潘红涛. 上海电力学院学报, 2010, No. 6, 567.

[11] 王敬. 科技风, 2010, No. 17, 47 .

[12］范铸法. [2020-8-9]. https://zh.wikipedia.org/wiki/\%E7\%AF\%84\%E9\%91\%84\%E6\%B3\%95.

[13] 青铜神树. [2020-08-09]. https://zh.wikipedia.org/wiki/\%E9\%9D \%92\%E9\%93\%9C\%E7\%A5\%9E\%E6\%A0\%91.

[14] 越王勾践剑. [2020-08-09]. https://zh.wikipedia.org/wiki/\%E8\%B6\%8A\%E7\%8E\%8B $\%$ E5\%8B $\%$ BE\%E8\%B7\%B5\%E5\%89\%91.

[15] 杨国庆. 中原文物, 1991, No. 2, 107.

[16] 乔爱梅. 学术研究, 2015, No. 2, 88 .

[17] 后德俊. 江汉考古, 1980, No. 1, 63.

[18] 龙泉剑. [2020-08-10]. https://baike.baidu.com/item/\%E9\%BE\%99\%E6\%B3\%89\%E5\%89\%91/5278879?fromtitle=\%E9\%BE\%99\%E6\%B3\% $89 \% \mathrm{E} 5 \% \mathrm{AE} \% 9 \mathrm{D} \% \mathrm{E} 5 \% 89 \% 91 \&$ fromid $=2463631$. 